\title{
Chronic Glucocorticoids Increase Hippocampal Vulnerability to Neurotoxicity under Conditions That Produce CA3 Dendritic Retraction But Fail to Impair Spatial Recognition Memory
}

\author{
Cheryl D. Conrad, Katie J. McLaughlin, James S. Harman, Cainan Foltz, Lindsay Wieczorek, Elizabeth Lightner, and \\ Ryan L. Wright \\ Department of Psychology, Arizona State University, Tempe, Arizona 85287-1104
}

\begin{abstract}
We previously found that chronic stress conditions producing CA3 dendritic retraction and spatial memory deficits make the hippocampus vulnerable to the neurotoxin ibotenic acid (IBO). The purpose of this study was to determine whether exposure to chronic corticosterone (CORT) under conditions that produce CA3 dendritic retraction would enhance CA3 susceptibility to IBO. Male Sprague Dawley rats were chronically treated for $21 \mathrm{~d}$ with CORT in drinking water $(400 \mu \mathrm{g} / \mathrm{ml})$, and half were given daily injections of phenytoin (40 $\mathrm{mg} / \mathrm{kg}$ ), an antiepileptic drug that prevents CA3 dendritic retraction. Three days after treatments stopped, IBO was infused into the CA3 region. Conditions producing CA3 dendritic retraction (CORT and vehicle) exacerbated IBO-induced CA3 damage compared with conditions in which CA3 dendritic retraction was not observed (vehicle and vehicle, vehicle and phenytoin, CORT and phenytoin). Additionally, spatial recognition memory was assessed using the Y-maze, revealing that conditions producing CA3 dendritic retraction failed to impair spatial recognition memory. Furthermore, CORT levels in response to a potentially mild stressor (injection and Y-maze exposure) stayed at basal levels and failed to differ among key groups (vehicle and vehicle, CORT and vehicle, CORT and phenytoin), supporting the interpretations that CORT levels were unlikely to have been elevated during IBO infusion and that the neuroprotective actions of phenytoin were not through CORT alterations. These data are the first to show that conditions with prolonged glucocorticoid elevations leading to structural changes in hippocampal dendritic arbors can make the hippocampus vulnerable to neurotoxic challenges. These findings have significance for many disorders with elevated glucocorticoids that include depression, schizophrenia, Alzheimer's disease, and Cushing's disease.
\end{abstract}

Key words: corticosterone; dendritic arborization; depression; exploration; glucocorticoid; hippocampus; ibotenic acid; learning and memory; neuronal death; rat; recognition memory; stress

\section{Introduction}

Chronic stress can be particularly debilitating to the hippocampus, a limbic brain region involved in spatial memory and the regulation of glucocorticoids [such as corticosterone (CORT)]. Chronic stress and glucocorticoids reduce hippocampal dendritic complexity (Watanabe et al., 1992a; Magariños and McEwen, 1995a; Conrad et al., 1999b; Kleen et al., 2006: McLaughlin et al., 2007) and can even cause hippocampal cell death (Landfield et al., 1978; Uno et al., 1989; Sapolsky, 2005). Hippocampal dendritic remodeling in the CA3 region is commonly studied after chronic stress or glucocorticoid administration because the

Received March 15, 2007; revised June 1, 2007; accepted June 20, 2007.

This work was supported by National Institutes of Health Grant MH64727 (C.D.C.) and by funds from the Arizona State University (ASU) Office of the Provost through the School of Life Sciences Enrichment Programs (J.S.H., C.F., L.W., and E.L.). Data were presented at the 2007 Annual Meeting of the Society for Neuroscience. We especially thank Sarah Baran, Jonathan Kleen, Sanford Braver, Edward Castañeda, and ASU Barrett Honors College.

Correspondence should be addressed to Dr. Cheryl D. Conrad, Department of Psychology, Arizona State University, Box 1104, Tempe, AZ 85287-1104. E-mail: conradc@asu.edu.

D0I:10.1523/JNEUROSCI.2121-07.2007

Copyright $\odot 2007$ Society for Neuroscience $\quad$ 0270-6474/07/278278-08\$15.00/0
CA3 region is among the first hippocampal areas to show dendritic restructuring (Woolley et al., 1990; Magariños and McEwen, 1995a), which can reverse to the prestress condition after cessation of chronic stress or glucocorticoid treatment (Conrad et al., 1999b). However, CA3 dendritic retraction may increase vulnerability to concurrent life events. For example, chronic stress conditions that create CA3 dendritic retraction may also exacerbate CA3 damage after a neurotoxin challenge [such as ibotenic acid (IBO)], leading to the hypothesis that dendritic retraction may represent compromised neurons (Conrad et al., 2004).

The main purpose of this study was to determine whether a history of chronic glucocorticoid elevations mediate the chronic stress exacerbation of CA3 damage after neurotoxin challenge. Chronic stress produces hippocampal dendritic retraction through the actions of glucocorticoids (Woolley et al., 1990; Watanabe et al., 1992b; Magariños and McEwen, 1995b). If dendritic retraction contributes to hippocampal damage after neurotoxin challenge, then producing dendritic retraction using glucocorticoids should also exacerbate neurotoxin-induced hippocampal 
Exp. 1: Chronic CORT \& Phenytoin on IBO-Induced CA3 Damage

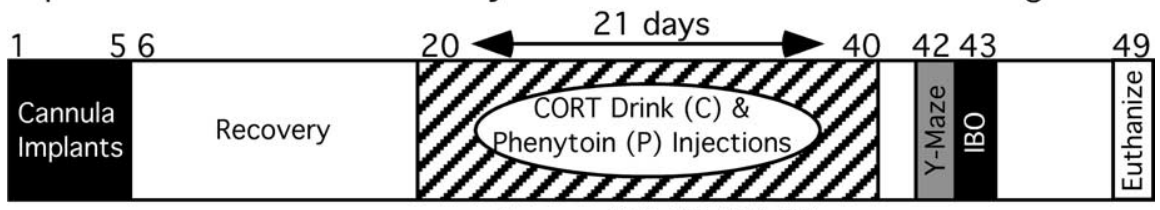

Groups: VV, VP, CV, CP

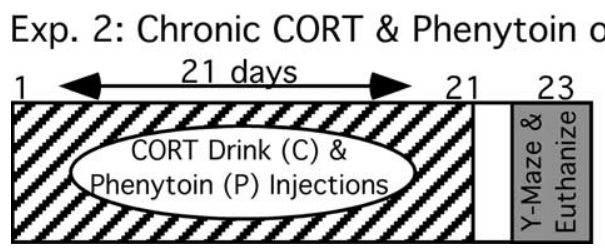

Figure 1. Experimental timeline. Experiment 1 investigated the effects of chronic glucocorticoid (CORT) and phenytoin on Y-maze performance and susceptibility to IB0. Experiment 2 investigated the effects of chronic glucocorticoid and chronic phenytoin on CA3 dendritic complexity.

damage. Moreover, the antiepileptic drug phenytoin (Dilantin) blocks CA3 dendritic retraction caused by chronic stress or glucocorticoids without interfering with other physiological effects of chronic stress, such as changes in adrenal or body weight (Watanabe et al., 1992b; Magariños and McEwen, 1995b). Thus, we hypothesized that conditions producing CA3 dendritic retraction would exacerbate neurotoxin-induced hippocampal damage.

A second purpose of this study was to investigate the effects of chronic glucocorticoid elevations on spatial recognition memory. Studies investigating the effects of chronic glucocorticoid exposure on spatial ability have reported mixed results, with some studies showing intact spatial memory under conditions that should produce CA3 dendritic retraction (Luine et al., 1993; Magariños et al., 1998; Coburn-Litvak et al., 2003), whereas other studies show spatial memory deficits (Dachir et al., 1993; McLay et al., 1998). Therefore, the following study included spatial memory assessment after a history of long-term glucocorticoid and phenytoin exposure.

\section{Materials and Methods}

Subjects. The Arizona State University Institutional Animal Care and Use Committee approved all procedures, in accordance with the applicable portions of the Animal Welfare Act and the Guide for the Care and Use of Laboratory Animals by the Department of Health and Human Services. All efforts were made to minimize the number of subjects used and any potential suffering. Male Sprague Dawley rats (Charles River, Hartford, CT) were single-housed (experiment 1 because of the cannula implants) or pair-housed (experiment 2) in temperature- and light-controlled chambers ( $12 \mathrm{~h}$ schedule, lights off at 6:00 A.M.). On arrival, rats weighed $\sim 225-260 \mathrm{~g}$ and were allowed to acclimate to the facilities for 1 week before being randomly assigned to the experimental groups, with control and CORT-treated rats housed in separate, but identical, housing chambers.

Handling procedures. Rats were handled daily to acclimate them to transport and handling procedures for Y-maze testing and IBO infusion. Handling involved transporting several home cages to a location within the laboratory, picking up a rat, and gently holding it to restrict head movement for those with cannula implants. Handling sessions lasted $\sim 1-2$ min for each rat.

Chemicals. Phenytoin (40 mg/kg; Sigma, St. Louis, MO) was made fresh every few days by being dissolved in propylene glycol and sonicated daily before filling syringes (Reagan et al., 1999). CORT (ICN Biomedicals, Costa Mesa, CA) drinking solution $(400 \mu \mathrm{g} / \mathrm{ml})$ was prepared by dissolving CORT $(16.6 \mathrm{mg} / \mathrm{ml})$ in $100 \%$ ethanol stock solution and then diluting this stock solution to the final concentration with tap water (2.4\% final ethanol concentration). IBO (Sigma) was prepared fresh on the day it was used and diluted to a concentration of $1 \mu \mathrm{g} / \mu \mathrm{l}$ using PBS (0.1 M, pH 7.4).

$Y$-maze apparatus. The Y-maze was used to assess spatial memory, as described by Conrad et al. (1996), and consisted of three equilaterally intersecting Plexiglas arms $(58 \mathrm{~cm}$ long $\times 19$ $\mathrm{cm}$ wide $\times 38 \mathrm{~cm}$ high). Spatial cues included geometric designs on the surrounding walls. To prevent utilization of odor cues, two identical Y-mazes were exchanged between trials, and the corncob bedding on floor of the Y-mazes was mixed between trials. The Y-mazes were carefully positioned in the same location as the previous setup.

Experiment 1 (IBO): effect of CORT drink history and daily phenytoin injections on IBO-induced CA3 damage and spatial memory

The purpose of this study was to investigate the effects of chronic CORT and phenytoin on hippocampal CA3 susceptibility to excitotoxin IBO challenge and Y-maze performance. Figure 1 summarizes the timeline and procedures performed in experiment 1 . Rats were implanted with guide cannulas before the start of the experiment to avoid confounds that could have been generated from surgical stress after chronic CORT and phenytoin administration. In experiment 1 , rats were singlehoused to facilitate healing and reduce the chance of infection from the surgical implants.

Surgery (days 1-5). Rats were implanted with guide cannulas before the start of the experiment using aseptic stereotaxic surgical procedures. Rats were pretreated with atropine sulfate $(0.1 \mathrm{cc}$ of $0.54 \mathrm{mg} / \mathrm{ml}$, i.p. $) 10$ min before they were deeply anesthetized with sodium pentobarbital (55 $\mathrm{mg} / \mathrm{kg}$, i.p.). A stereotaxic frame (Kopf Instruments, Tujunga, CA) held the head securely, and guide cannulas (26 ga and $4 \mathrm{~mm}$ long below pedestal; Small Parts, Miami Lakes, FL) were lowered until the pedestal touched the skull at coordinates targeting the CA3 region of the hippocampus (anteroposterior, $-3.6 \mathrm{~mm}$; mediolateral, $\pm 3.3 \mathrm{~mm}$; interaural line, 0$)$. The guide cannulas were fixed into place with four skull screws $(3.5 \mathrm{~mm}, 8 \mathrm{~mm}$ tread diameter; Lomat, Montreal, Quebec, Canada) and cranioplastic cement (Stoelting, Wood Dale, IL). Stainless steel stylets (33 ga, $4 \mathrm{~mm}$ long below pedestal; Small Parts) were placed into the guide cannulas to prevent occlusion. Animals were allowed 10-14 d for recovery.

Chronic CORT and phenytoin administration (days 20-40). CORT $(400 \mu \mathrm{g} / \mathrm{ml})$ was added in the drinking water and dyed with red food coloring to distinguish it from tap water. Controls were given tap water with ethanol vehicle $(2.4 \%)$, which was dyed with green food coloring. Half of the rats in each group were given daily injections of phenytoin (40 $\mathrm{mg} / \mathrm{kg}$, s.c.), whereas the remaining rats received vehicle $(1 \mathrm{ml} / \mathrm{kg}$ propylene glycol). Body weights were measured on days 20 and 40, representing the start and end of the CORT and phenytoin manipulations.

Y-maze testing (day 42). Two days after the CORT drinking solution and phenytoin injections ended, rats were trained in the Y-maze with a $4 \mathrm{~h}$ delay before testing. Rats were tested in random order, and two rats were tested in separate Y-mazes at the same time. The rats were placed in an arm of the Y-maze (start arm) with one of the arms blocked off (novel arm) and were allowed to explore the start arm and remaining arm (other arm) for $15 \mathrm{~min}$. Rats were then removed from the maze, the novel arm was unblocked, the Y-mazes were swapped, and the bedding was mixed. After a $4 \mathrm{~h}$ delay, rats were placed back in the original spatial location of the start arm and allowed to explore all arms freely for $5 \mathrm{~min}$. Because rats tend to explore novel environments, functional memory was measured as the number of entries into the novel arm compared with the other arm. An entry was quantified when all four paws entered an arm. Poor memory would lead to relatively equal entries into the novel and other arms. Behavior was videotaped for quantification at a later date. For a detailed review of this Y-maze protocol, see Conrad (2006).

IBO infusion (day 43). Rats were transported to one of the laboratory 
rooms where they were previously handled and acclimated. An investigator gently held the rat, removed the dummy cannula from the guide cannula, and inserted an infusion cannula ( $33 \mathrm{ga}, 5 \mathrm{~mm}$ below pedestal to extend $1 \mathrm{~mm}$ below the guide cannula). The infusion cannula was attached via polyethylene 20 tubing (Dow Corning, Midland, MI) to 10.0 $\mu l$ Hamilton (Reno, NV) syringes mounted on an infusion pump (Harvard Apparatus, South Natick, MA). IBO $(1 \mu \mathrm{g} / 1 \mu \mathrm{l})$ was infused unilaterally for $2 \mathrm{~min}$, and the cannula was left in place for an additional $2 \mathrm{~min}$ to allow the neurotoxin to diffuse. PBS $(0.1 \mathrm{M})$ was infused on the contralateral side, with each side counterbalanced among rats. The dose of IBO was chosen based on pilot work for our previous study (Conrad et al., 2004) that showed higher doses producing a ceiling effect in creating $100 \%$ damage at the infusion site. After bilateral infusions, rats were returned to their home cages.

Perfusion and histology (day 49). Observing IBO-induced cell death and microglia proliferation requires at least a $7 \mathrm{~d}$ wait after infusion (DeGiorgio et al., 2002). Consequently, $7 \mathrm{~d}$ after IBO infusion, rats were overdosed with sodium pentobarbital (100 mg/kg, i.p.). Rats were perfused transcardially with $\sim 60 \mathrm{cc}$ of $0.1 \mathrm{M}$ PBS and $120 \mathrm{cc}$ of $4 \%$ formalin in $0.1 \mathrm{M}$ PBS. Brains were removed and stored in $4 \%$ formalin until tissue sectioning.

Two days before sectioning, brains were placed in $30 \%$ sucrose for cryoprotection. Brains were cut $(30 \mu \mathrm{m})$ in the frontal plane using a Micron (San Marcos, CA) cryostat at $-22^{\circ} \mathrm{C}$ and mounted on gelatinchromium subbed slides until the dorsal hippocampus was exhaustively sectioned. Lost sections, if any, were noted for accurate reconstruction. Brain sections were stained with $0.1 \%$ cresyl violet and coverslipped with Permount (VWR Scientific, West Chester, PA) after submersion in ethanol dilutions and a xylene substitute (CitriSolv, 15 min; Fisher Scientific, Pittsburgh, PA).

Histological quantification of IBO damage. Sections were coded and remained sealed until the analysis was complete. Details of damage quantification were described previously (Conrad et al., 2004). Briefly, the section containing the needle tip was identified at a magnification of $100 \times$ using an Olympus (Tokyo, Japan) microscope (model BX50) and referred to as the infusion site. Damage to the CA3 region was determined by identifying the absence of CA3 pyramidal cells in the subregion $\mathrm{CA} 3 \mathrm{c}$, which corresponds to the same region that has been reported to express CA3 dendritic retraction after chronic stress or CORT exposure. These CA3 cells are localized within a thin band, called the stratum pyramidale, and this band can be measured in length or distance. The extent of this damage was then quantified using a calibrated grid $(0.0125$ inch divisions; total length, $5 / 8$ inch) in the ocular lens of the microscope. The distance representing CA 3 cell damage was divided by the total CA3 distance and converted into a percentage. Sections in the anterior and posterior planes of the infusion site were averaged to determine the percentage of CA3 damage at each $120 \mu \mathrm{m}$ increment from the infusion site. Therefore, four $30 \mu \mathrm{m}$ sections were averaged in the posterior and anterior directions of the infusion site to provide one value for each $120 \mu \mathrm{m}$ increment. In the few cases that sections were lost, the distance of the lost section was skipped, and the average damage of the remaining sections was calculated.

To analyze the histological data, the four groups of rats [VV, VP, CV, and $\mathrm{CP}$ (V, vehicle; $\mathrm{P}$, phenytoin; $\mathrm{C}, \mathrm{CORT})]$ were matched according to (1) the location of the infusion site and (2) the percentage of damage at the site of infusion. The infusion site location was matched using its position in all three planes: anteroposterior, mediolateral, and dorsoventral. The amount of damage at the infusion site had to be within $10 \%$ for sections to be matched. Matching groups across these dimensions was necessary because IBO damage varies depending on guide cannula location.

\section{Experiment 2 (Golgi): effect of CORT drink history and daily} phenytoin injections on CA3 dendritic morphology

The purpose of this study was to confirm that chronic CORT produced CA3 dendritic retraction and that daily phenytoin injections prevented CA3 dendritic retraction.

Chronic CORT and phenytoin administration (days 1-21). Procedures for providing CORT in the drinking water were the same as in experi- ment 1 . Body weights were measured on days 1 and 21 , representing the start and end of the CORT and phenytoin manipulations (Fig. 1). Rats in this experiment were exposed to the Y-maze as described for experiment 1 but were given injections of sesame oil ( $1 \mathrm{ml} / \mathrm{kg}$, s.c.) $\sim 40 \mathrm{~min}$ before they were killed, when trunk blood was collected for subsequent CORT determination. Sesame oil injection is commonly performed in experiments administering lipid-soluble steroids (Conrad et al., 1999a), and the injection process can produce a mild stress response (Selye, 1984).

CORT assay. Blood was centrifuged for $30 \mathrm{~min}$ at $4000 \mathrm{rpm}$ using a Heraeus centrifuge (model Megafuge 1.0R; VWR Scientific). Serum was removed and stored at $-70^{\circ} \mathrm{C}$. Samples were diluted 1:10 and processed in duplicate. Final values for each subject were averaged and represented as micrograms per deciliter. CORT levels were determined using an enzyme immunoassay kit (catalog \#026-AC-14F1; American Laboratory Products, Windham, NH). Antibody cross-reactivity to other steroids did not exceed $0.05 \%$. Optical density values were measured at $450 \mathrm{~nm}$ using a microplate reader (model LabSystems Multiskan RC; Fisher Scientific).

Histology for Golgi. Brains were quickly removed, frozen in 2-methylbutane, and processed according to the directions of a rapid Golgi staining kit (FD Neurotechnologies Consulting \& Services, Ellicott City, MD). Brains were cut in $100 \mu \mathrm{m}$ sections (Microtome HM $500 \mathrm{OM}$ Cryostat; $-30 / 32^{\circ} \mathrm{C}$ ) and, when placed on a slide, were firmly pressed by hand using Bibulous blotting paper (Fisher Scientific). Sections were processed until stained as directed by the Golgistain kit. Slides were kept in the dark to dry for 1-2 weeks and coverslipped with Permount mounting medium (Fisher Scientific).

For proper Golgi analysis, cells were chosen based on the following criteria: (1) the cell body and dendrites were fully impregnated; (2) the cell was relatively isolated from surrounding neurons; and (3) the cell was located in the $\mathrm{CA} 3 \mathrm{c}$ region of the hippocampus. A camera lucida drawing tube attached to an Olympus BX51 microscope was used to trace all neurons $(320 \times)$. Dendritic length was quantified using a Scion (Frederick, MD) Image Microcomputer Imaging Device Program attached to an Olympus BX50 microscope. CA3 neurons were further labeled as shortshaft (SS) or long-shaft (LS) depending on their relative location in the stratum pyramidale and proximal apical shaft length (Fitch et al., 1989). The apical dendrites of SS neurons in the CA3 region are intrinsically more complex than the apical dendrites of the LS neurons, and so the statistical analyses were performed to ensure that both cell types were equally represented in each rat. For a rat to be included in the analysis, the brains contained successfully stained neurons of both SS and LS categories, which were averaged separately before the two values for the SS and LS neurons were averaged to obtain one value for each rat. Dendritic length and branch points (number of dendritic bifurcations) were measured separately for the apical and basal sections of SS and LS neurons.

\section{Statistical analyses}

Parametric data were analyzed by ANOVA. The histological data were transformed using the equation for proportions, $2 \arcsin$ (square root of Y), which allows ANOVA to be performed (Cohen et al., 2003). Newman-Keuls post hoc tests or planned comparisons were performed when statistical significance was reached ( $p \leq 0.05$ ). Nonparametric data were analyzed by Wilcoxon tests. Data are represented by means \pm SEM. The inter-rater reliability was $97.3 \pm 1.0 \%$ for determining CA3 damage caused by IBO (experiment 1 ) and $97.0 \pm 1.03 \%$ for quantifying CA3 dendritic length (experiment 2).

\section{Results}

\section{Experiment 1 (IBO): effect of CORT drink history and daily phenytoin injections on IBO-induced CA3 damage and spatial memory}

Based on the brain section containing the needle tip, six pairings were made among the four groups (VV, VP, CV , CP) with similar needle tip placements and percentage of CA3 damage at the site of infusion. Of the original 62 rats implanted with cannulas, the final number of rats that had accurate cannula placement and percentage of CA3 damage at the infusion point that exceeded 


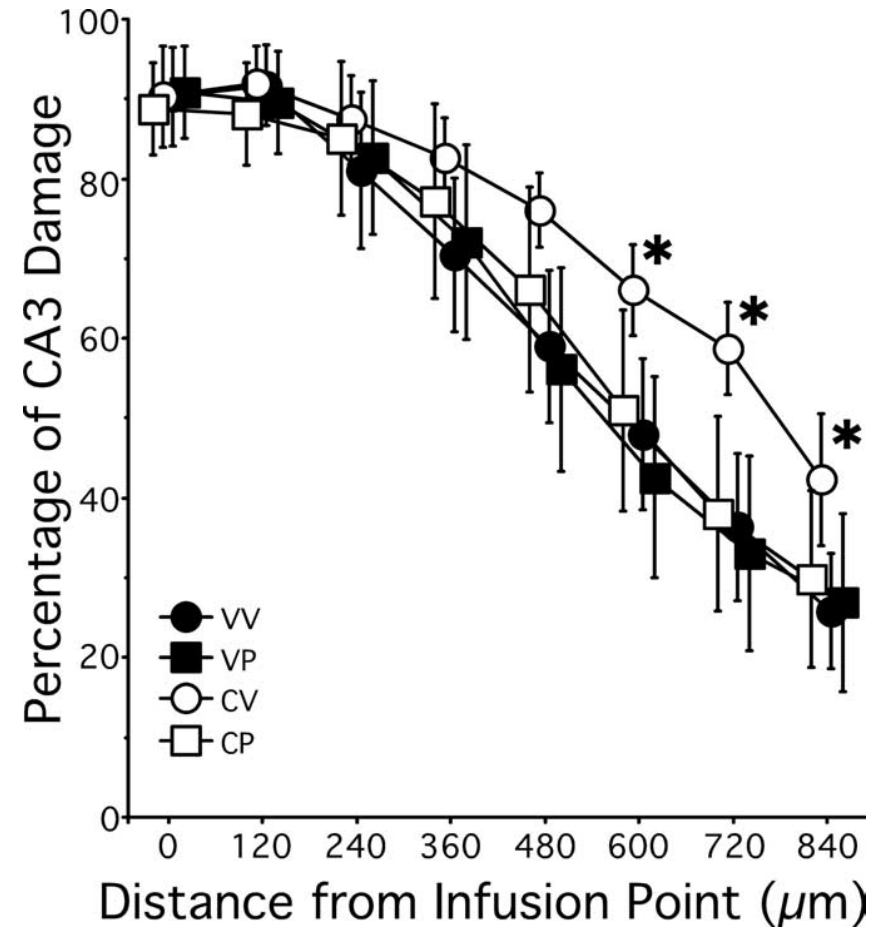

Figure 2. Percentage of CA3 hippocampal damage after IBO infusion. Rats chronically treated with CORT drinking water and daily injections of vehicle $(\mathrm{CV})$ showed more IBO-induced CA3 damage compared with control groups (VV, VP). In contrast, rats treated with CORT drinking water and daily phenytoin injections (CP) showed statistically similar levels of damage as the controls (VV, VP), indicating that phenytoin prevented the negative consequences of CORT on CA3 susceptibility to IBO-induced damage. ${ }^{*} p<0.05$ compared with CP. $n=6$ per group. Error bars indicate means \pm SEM.

50\% was as follows: VV, $n=7$; VP, $n=11$; CV , $n=9$; CP, $n=8$ (total of 35). The remaining rats $(n=27)$ were not included in the final analysis for the following reasons: cannula placements were inaccurate $(n=17)$, the percentage of CA3 damage at the needle tip failed to reach $50 \%(n=6)$, the tissue was mechanically damaged $(n=3)$, or the rat died $(n=1)$.

The percentage of CA3 damage caused by IBO infusion was significantly influenced by the history of rats drinking CORT solution, daily injections of phenytoin, and distance from the site of IBO infusion. For the first analysis, a $2 \times 2 \times 8$ mixed-factor ANOVA was performed for drinking-solution history (vehicle, CORT), daily drug injection (vehicle, phenytoin), and distance from the IBO infusion site $(0,120,240,360,480,600,720,840$ $\mu \mathrm{m})$. The analysis showed a significant effect of distance $\left(F_{(7,140)}\right.$ $=181.7 ; p<0.001)$ and a significant interaction for drinkingsolution history $\times$ distance $\left(F_{(7,140)}=3.57 ; p=0.001\right)$. For distance, a reduced percentage of CA3 damage was observed as the distance from the IBO infusion site increased. The percentage of CA3 damage at the infusion site $(0 \mu \mathrm{m})$ and at $840 \mu \mathrm{m}$ away from the infusion site averaged (for all groups) 90 and 30\%, respectively. For the drink $X$ distance interaction, the percentage of CA3 damage in the CORT groups $(\mathrm{CV}, \mathrm{CP})$ statistically differed from the vehicle groups (VV, VP) by $360 \mu \mathrm{m}$ from the infusion site (Fig. 2) $(p<0.05)$ and continued to significantly differ at the remaining distances $(480,600,720,840 \mu \mathrm{m})$. This increased susceptibility of the $\mathrm{CA} 3$ region to IBO infusion appeared to be predominately carried by the $\mathrm{CV}$ group, and so a separate mixedfactor ANOVA was performed using the CORT group (CV, CP) by distance $(360,480,600,720,840 \mu \mathrm{m})$. The ANOVA showed a significant effect of distance $\left(F_{(4,40)}=75.8 ; p<0.001\right)$ and sig- nificant interaction for group $\times$ distance $\left(F_{(4,40)}=2.56 ; p=\right.$ $0.05)$. This analysis revealed that IBO infusion in rats with a history of CORT drink and daily vehicle injections (CV) had significantly more CA3 damage compared with rats given CORT drink and daily phenytoin (CP). Importantly, phenytoin treatment in rats given CORT drink $(\mathrm{CP})$ prevented this exacerbation of CA3 damage by IBO.

To determine whether the infusion procedure would exacerbate damage in the rats given a history of CORT drink, the salineinfused side was inspected. Only two brains had minor CA3 damage; the average CA3 damage at the infusion site was $1.7 \pm 1.2 \%$.

\section{Y-maze}

Initial analyses were performed to determine the optimal minutes to assess spatial recognition memory because rats habituate quickly to the Y-maze. A mixed-factor ANOVA for drinkingsolution history (vehicle, CORT) and daily drug injection (vehicle, phenytoin) for total arm entries made during each minute ( 1 , $2,3,4,5)$ revealed a significant effect of minute $\left(F_{(3,228)}=29.6\right.$; $p<0.001)$. Rats made the most entries in minute 1 , which was significantly greater than all other minutes $(2,3,4,5 ; p<0.001$ for all four comparisons). Moreover, rats made significantly more entries in minute 2 compared with the remaining minutes $(3,4,5 ; p<0.01$ for all three comparisons). Minutes 3 and 4 were statistically similar. These analyses indicated that minutes 1 and 2 should be combined for further analyses because habituation in subsequent minutes can mask performance.

Spatial recognition memory was measured within each group by converting the number of entries made into each arm (novel, other, start) during minutes 1 and 2 into percentages of total entries. Wilcoxon analyses were used to compare the percentage of entries into the novel arm compared with the percentage of entries into the other arm. All groups made significantly more entries into the novel arm compared with the other arm (Fig. 3A).

A second analysis was performed to compare across groups by converting the percentage of entries into difference scores to obtain one value, which can range from $+100 \%$ (all entries were made into the novel arm) to $-100 \%$ (all entries were made into the other arm). Chance $(0 \%)$ reflects an equal number of entries into the novel and other arms. As can be seen in Figure $3 B$, all groups showed positive difference scores, supporting the Wilcoxon analyses that the rats entered the novel arm more than the other arm. Moreover, a three-way ANOVA performed on drinking-solution history (vehicle, CORT) and daily drug injection (vehicle, phenytoin) on the difference scores showed no significant effects. These findings show that novel arm recognition is unaltered by a history of chronic CORT or phenytoin.

To determine whether performance on the Y-maze was influenced by motivational or motor factors, the total number of entries made into the arms during minutes 1 and 2 were analyzed. A $2 \times 2$ ANOVA for drinking-solution history (vehicle, CORT) and daily drug injection (vehicle, phenytoin) was performed on total entries made during minutes 1 and 2. A significant main effect of drink history $\left(F_{(1,57)}=3.94 ; p=0.05\right)$ was found. Rats given CORT in their drinking water $(\mathrm{CV}, \mathrm{CP})$ made fewer total arm entries than rats given the vehicle drinking solution (VV, VP) (Fig. 3C). No other significant effects were observed.

Experiment 2 (Golgi): effect of CORT drink history and daily phenytoin injections on CA3 dendritic morphology

To determine the complexity of CA3 dendritic arbors in this chronic CORT and phenytoin paradigm, a separate group of rats was given CORT drink (or vehicle) and daily phenytoin (or ve- 

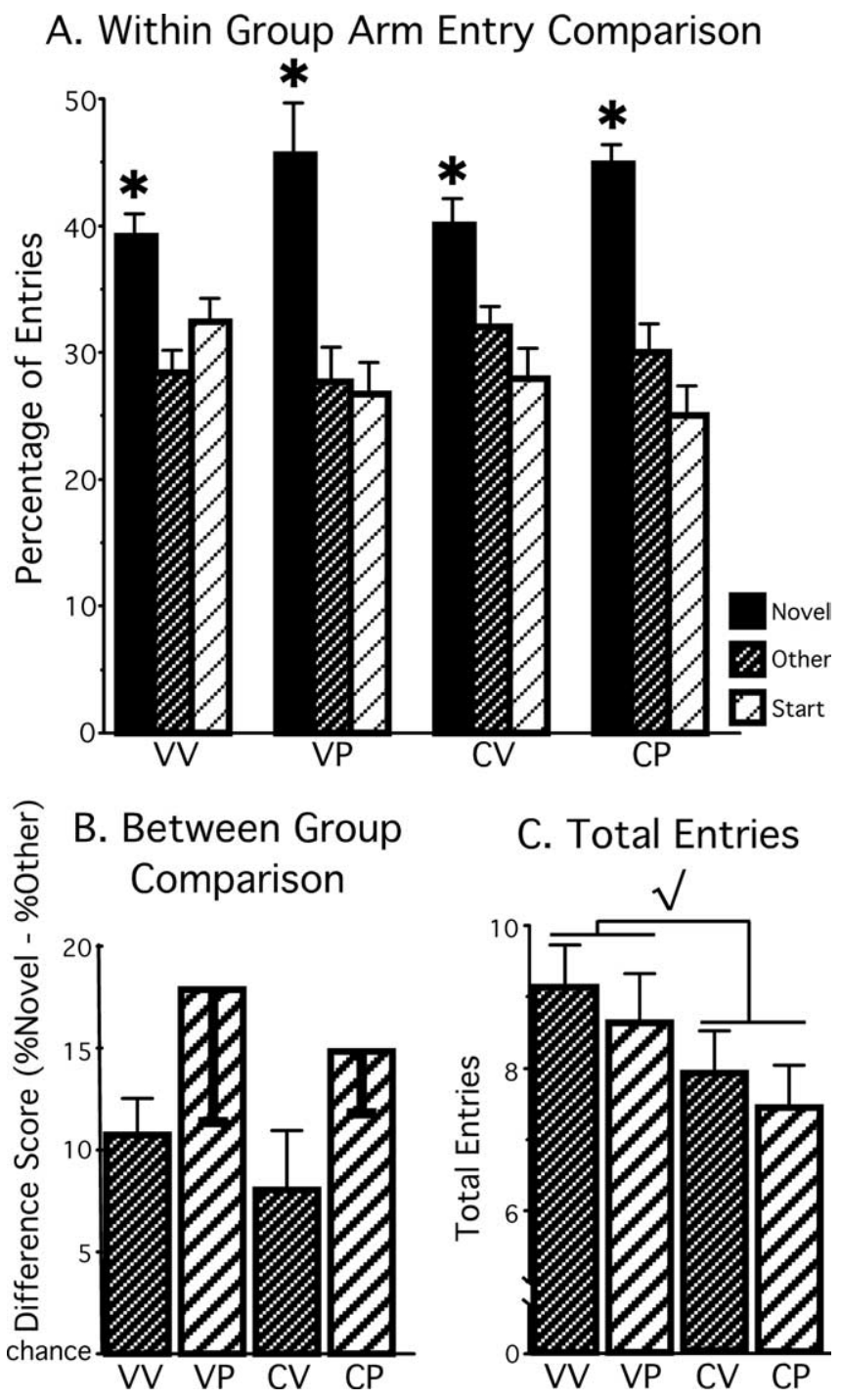

Figure 3. Influence of chronic CORT and phenytoin on Y-maze performance. $A$, All groups showed a significantly higher percentage of entries into the novel arm than the other arm, indicating that recognition of the novel arm was intact. ${ }^{*} p<0.05$ novel versus other arm using Wilcoxon analysis. $\boldsymbol{B}$, Animals given injections of phenytoin showed higher positive difference scores than groups given injections of vehicle, which shows that phenytoin improves novel arm recognition in the Y-maze above the standard performance and that novel arm recognition is unaltered by a history of chronic CORT. C, Rats given CORT in their drinking water made fewer total arm entries than did rats given the vehicle drinking solution. Error bars indicate means \pm SEM.

hicle) injections. Of the original 40 rats, 30 had between four and eight clearly stained and easily identifiable neurons localized within the CA3 region with representations of both short- and long-shafted neurons. The final number of rats within each condition was as follows: VV, $n=8$; VP, $n=7$; CV,$n=9$; CP, $n=6$. The average number of neurons per rat was $5.9 \pm 0.5(\mathrm{VV}), 6.1 \pm$ $0.7(\mathrm{VP}), 5.9 \pm 0.5(\mathrm{CV})$, and $6.7 \pm 0.3(\mathrm{CP})$.

CORT in the drinking solution decreased apical CA3 branch points, which was nearly prevented with daily phenytoin injections. A one-way ANOVA for the number of apical branch points was significant for group (VV, VP, CV, CP) $\left(F_{(3,26)}=3.20 ; p<\right.$ 0.05). Planned comparisons revealed that CORT in the drinking water $(\mathrm{CV})$ reduced the apical branch point complexity of CA3 neurons compared with vehicle controls (VV; $p=0.01$ ). Daily phenytoin injections improved the apical branch complexity of

\section{Apical Dendrites}
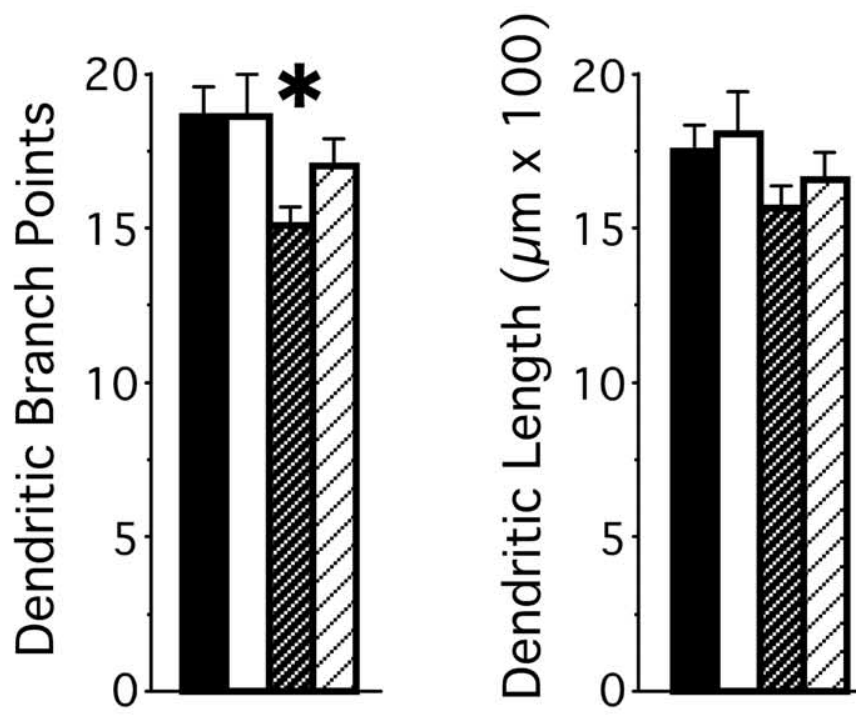

\section{Basal Dendrites}

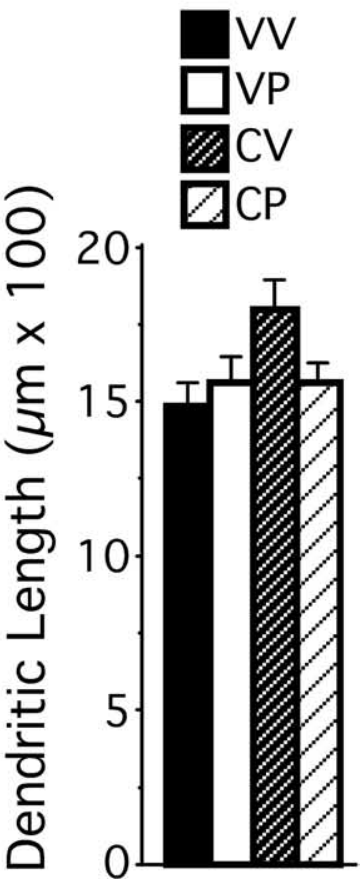

Figure 4. Quantification of $\mathrm{CA} 3$ apical and basal dendritic morphology. Treating rats with chronic CORT in the drinking water and daily vehicle injections (CV) reduced apical $C A 3$ dendritic branch points but did not significantly alter apical $C A 3$ dendritic length. Basal CA3 dendritic branch points and length were unaffected by chronic CORT and daily phenytoin treatment (CP). ${ }^{*} p<0.05$ compared with VV. $n=6-9$ per group. Error bars indicate means \pm SEM.

CORT-treated rats (CP) because CA3 branch point number of CP was statistically similar to VV and VP. However, CV and CP were statistically similar, indicating that phenytoin produced moderate improvements in CA3 apical branch points in CORTtreated rats (Figs. 4, 5). A one-way ANOVA for CA3 apical dendritic length showed a similar but nonsignificant pattern as was observed for the apical branch points $\left(F_{(3,26)}=1.24, p>0.05\right)$. For the basal dendritic region, a one-way ANOVA did not reach significance for branch point number $\left(F_{(3,26)}=2.60 ; p=\mathrm{NS}\right)$ or length $\left(F_{(3,26)}=2.84 ; p=\mathrm{NS}\right)$.

Trunk blood was collected $\sim 40$ min after sesame oil injections 


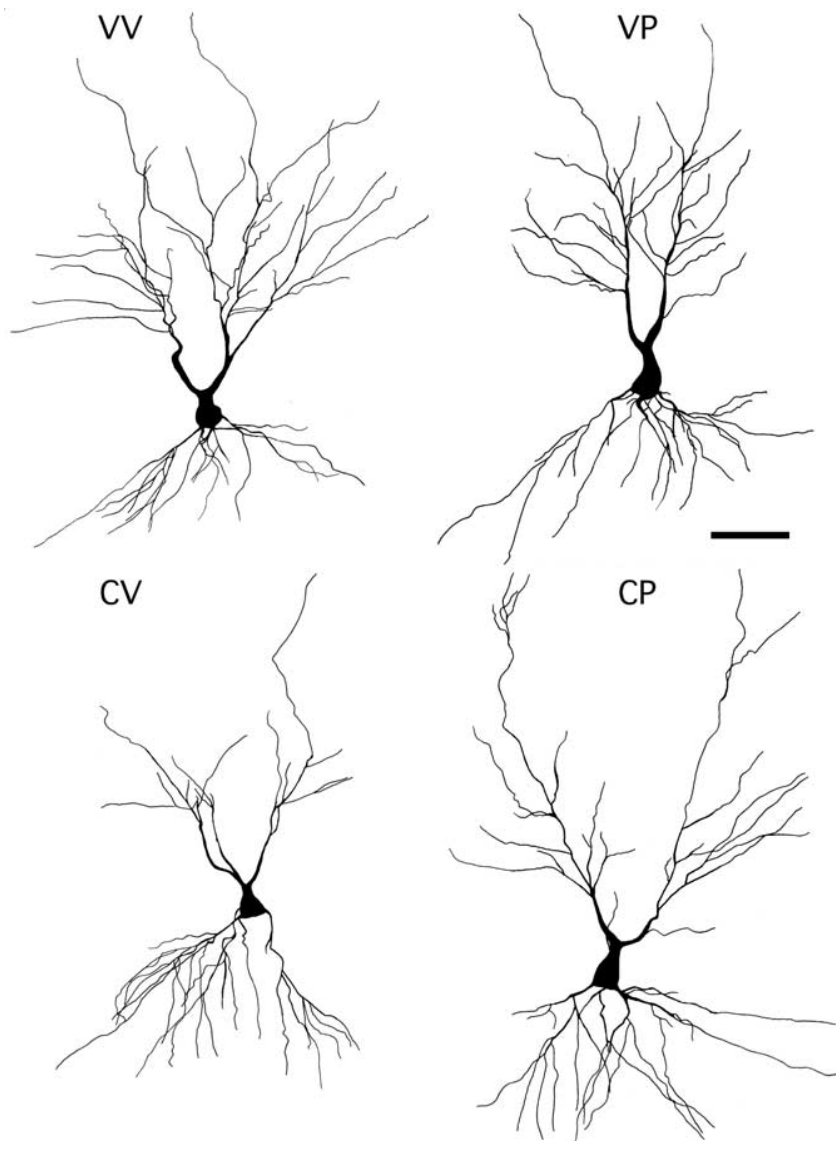

Figure 5. Representations of Golgi-stained CA3 neurons. Tracings were made using a camera lucida drawing tube $(320 \times)$. Note the reduced apical CA3 dendritic arbors of the CV condition relative to the other treatments.

for serum CORT determination. A $2 \times 2$ ANOVA for drinkingsolution history (vehicle, CORT) and daily drug injection (vehicle, phenytoin) was performed on serum CORT, revealing significant effects for history $\left(F_{(1,36)}=6.22 ; p<0.05\right)$, $\operatorname{drug}\left(F_{(1,36)}=\right.$ $11.99 ;<0.005)$, and the interaction between history and drug $\left(F_{(1,36)}=6.95 ; p<0.05\right)$. The significant main effects were predominately carried by the VP group (Table 1), which showed significant elevations of serum CORT $(9.5 \mu \mathrm{g} / \mathrm{dl})$ compared with the basal CORT levels of VV $(0.87 \mu \mathrm{g} / \mathrm{dl}), \mathrm{CV}(1.08 \mu \mathrm{g} / \mathrm{dl})$, and $\mathrm{CP}(2.35 \mu \mathrm{g} / \mathrm{dl})$. The most critical finding is that the serum CORT levels of the rats exposed to a history of chronic CORT (CV, CP) were statistically similar to each other and to the CORT levels of the vehicle controls (VV).

\section{Experiments 1 and 2: body weight}

Slow body weight gain is a reliable indicator of the effectiveness of long-term CORT treatment in male rats. In these two experiments, a history of CORT in the drinking water consistently reduced body weight gain. For experiment 1 , a $2 \times 2$ ANOVA for drinking-solution history (vehicle, CORT) and daily drug injection (vehicle, phenytoin) was performed on the net body weight gain over the $21 \mathrm{~d}$ that CORT was provided in the drinking water. A significant effect was observed for drinking-solution history $\left(F_{(1,58)}=70.1 ; p<0.0001\right)$ and interaction between drinkingsolution history and daily drug injection $\left(F_{(1,58)}=5.56, p<\right.$ $0.05)$. The VP group gained significantly more weight compared with the remaining groups (VV, CV, CP). Moreover, VV gained significantly more weight than both the groups given CORT in the drinking water $(\mathrm{CV}, \mathrm{CP})$, and both of these groups with a history of CORT in their drinking water showed statistically similar body weight gain (Table 1). For experiment 2, a one-way ANOVA for body weight gain was significant $\left(F_{(1,36)}=50.60 ; p<\right.$ $0.0001)$. The rats given CORT in their drinking water $(\mathrm{CV}, \mathrm{CP})$ showed statistically reduced body weight gain compared with rats given vehicle (VV, VP). Moreover, all CORT-treated rats had statistically similar levels of body weight gain, and the vehicletreated rats showed statistically similar levels of body weight gain (Table 1). These findings show that CORT in the drinking water produced a consistent reduction in body weight gain compared with vehicle-treated rats.

\section{Discussion}

These findings support the hypothesis that conditions producing CA3 dendritic retraction make the hippocampal CA3 region vulnerable to a neurotoxic challenge and that CA3 dendritic retraction may represent compromised neurons. We previously demonstrated that chronic stress exacerbates IBO-induced CA3 damage (Conrad et al., 2004). In the current study, we show that chronic elevation of glucocorticoids (i.e., CORT) can also increase IBO-induced CA3 damage, which suggests that the exacerbation of CA3 damage by chronic stress may be mediated by long-term glucocorticoid elevations. Moreover, the current study demonstrates that conditions unique to producing CA3 dendritic retraction are necessary for hippocampal vulnerability because enhanced IBO-induced CA3 damage was present in rats treated similarly as those showing CA3 dendritic retraction. In contrast, IBO did not exacerbate CA3 damage in chronic CORT-treated rats administered daily phenytoin (CP), which blocked CA3 dendritic retraction (experiment 2). Previous studies have shown that hippocampal susceptibility to neurotoxic and metabolic challenge is enhanced when glucocorticoids are elevated in close temporal proximity to the challenge (Sapolsky, 1985; Sapolsky and Pulsinelli, 1985; Sapolsky et al., 1988; Stein-Behrens et al., 1994). These findings are the first to show that conditions elevating glucocorticoids for prolonged durations to cause structural changes in hippocampal dendritic arbors can make the hippocampus vulnerable to neurotoxic challenges when glucocorticoids are unlikely to be elevated during the neurotoxic challenge.

To study the history of chronic glucocorticoid exposure on the hippocampus, the current study carefully avoided manipulations that would have elevated glucocorticoids during IBO infusion. First, cannulas were implanted before the glucocorticoid manipulation to allow IBO infusion in alert rats. Second, rats were acclimated to the infusion process to minimize potential stress from the procedure. Third, IBO infusion occurred $3 \mathrm{~d}$ after the chronic glucocorticoid exposure ended to allow metabolism of exogenous glucocorticoids, and thereby allowing the changes from the history of glucocorticoid exposure to be studied. Moreover, the reduced body weight gain and the atrophied CA3 apical dendritic arbors of the chronic glucocorticoid-treated rats showed that chronic glucocorticoid treatment in the drinking water was successful. These data indicate that glucocorticoids were unlikely to have been elevated during IBO infusion and that chronic glucocorticoid exposure was successfully administered.

Additional findings provide compelling support that CORT elevations during IBO infusion were unlikely to have mediated the enhanced hippocampal damage under the current conditions. Experiment 2 showed that sesame oil injections and Y-maze exposure did not elevate glucocorticoids 40 min later when endogenous glucocorticoids typically peak in response to a stressor (compare CV and CP with VV). Two critical findings 
were observed: (1) key groups (VV, CV, $\mathrm{CP})$ maintained basal levels of endogenous CORT secretion despite exposure to a potentially mild stressor and (2) rats exposed to history of chronic CORT (CV, CP) exhibited statistically similar CORT levels as control rats (VV). These results show that our rats successfully adapted to handling procedures and that phenytoin does not exert its protective effects via altering the hypothalamic-pituitary-adrenal (HPA) axis. Moreover, these findings highlight important differences between chronic stress and chronic CORT exposure. Chronic stress activates the HPA axis, whereas chronic CORT administration provides powerful negative feedback on the HPA axis. One consequence is that chronic stress alters the HPA axis in such a way that a heterotypic stressor (a novel unique acute stressor) leads to a potentiated CORT response, whereas a homotypic stressor (a familiar repeated stressor) leads to an attenuated CORT response (Wright et al., 2006) (for synopsis, see Dallman et al., 2000). Neither outcome was observed in the current study because the endogenous CORT levels were similar among key groups. Therefore, the exacerbation of IBO-induced damage by CORT history was unlikely to have been mediated by altered HPA axis response at the time of infusion.

As was done in our previous study (Conrad et al., 2004), great care was taken to deliver the appropriate amount of IBO and to match brains based on the location of the IBO infusion site. The selected dose of IBO was sufficient to kill cells without creating a ceiling effect or diffusion of IBO into regions outside of the CA3 region (Jarrard, 1989). Moreover, the side infused with saline showed negligible damage, which was similar among treatments (VV, VP, CV , CP). For the IBO infusion site, coded groups were matched based on the cannula placement at the site of infusion and the percentage of damage at the infusion site. As shown in Figure 2, all groups (VV, VP, CV, CP) showed statistically similar levels of CA3 damage at the site of IBO infusion $(0 \mu \mathrm{m})$, indicating that the matching process was not biased. Also, inter-rater reliability was very high, and because data are represented as a percentage of damage, it is unlikely that different investigators or unequal tissue shrinkage created a bias. These data show that a history of chronic glucocorticoid exposure exacerbated IBOinduced CA3 damage as opposed to artifacts of the procedure.

Another important finding was that spatial recognition memory was functional under conditions in which chronic glucocorticoid exposure was sufficient to produce CA3 dendritic retraction. All groups showed a preference for the novel arm by entering it more often than the other arm. Moreover, the use of intramaze cues was prevented by mixing the bedding on the floor of the maze between trials and swapping the mazes so that the rats were returned to the same location, but in a different, identical maze. Therefore, rats had to use cues located outside the maze to successfully navigate. However, CORT-treated rats (CV, CP) showed reduced motivation to explore, as indicated by fewer entries into all arms overall. Nonetheless, reduced motivation to explore did not impair spatial recognition memory because all groups entered the novel arm more than the other arm. Importantly, rats in the CV and CP conditions performed similarly and as well as rats in the VV and VP groups, which supports the interpretation that all groups showed functional spatial recognition memory despite conditions that produced CA3 dendritic retraction.

The finding that chronic glucocorticoids failed to impair spa- tial recognition memory despite CA3 dendritic retraction is consistent with several studies (Luine et al., 1993; Bodnoff et al., 1995; Coburn-Litvak et al., 2003). Studies that have found spatial learning and memory deficits after chronic glucocorticoid exposure differ from the present research. These studies used a protocol that may have produced neuron loss, and/or glucocorticoid treatment continued during training and testing, which combines the chronic and acute actions of glucocorticoids on performance (Bardgett et al., 1994, 1996; Dachir et al., 1995; Endo et al., 1996; McLay et al., 1998; Ramos-Remus et al., 2002). Indeed, CoburnLitvak et al. (2003) found spatial memory deficits when chronic glucocorticoid treatment extended to $56 \mathrm{~d}$. These findings support the interpretation that CA3 dendritic retraction is not responsible for spatial learning and memory deficits (Conrad, 2006).

Several findings support the interpretation that dendritic retraction may contribute to the mechanism that makes the hippocampus vulnerable to neurotoxic challenge: (1) hippocampal damage was exacerbated under conditions when CA3 dendritic retraction was found or expected (current study; Conrad et al., 2004) but not (2) when dendritic retraction was blocked (current study) or (3) when stress was too brief to produce dendritic retraction (Conrad et al., 2004). The importance of these findings is that the hippocampus may be vulnerable to a variety of compromising situations that follow chronic stress or glucocorticoid elevations, even when glucocorticoid elevations have subsided. Although this report used a neurotoxin challenge, hippocampal neurons may be susceptible to damage after chronic glucocorticoid exposure in response to metabolic challenges, ischemia, hypoxia, and hypoglycemia.

Glucocorticoid-induced susceptibility to brain damage is important because several human conditions involve chronic glucocorticoid elevations. Individuals with Cushing's disease, depression, Alzheimer's disease, and schizophrenia with elevated glucocorticoids may be susceptible to hippocampal damage (McEwen 2000; for review, see de Kloet et al., 2005). Some aged individuals have difficulty regulating the HPA axis and exhibit slower CORT recovery from stressors and higher basal CORT levels (Nichols et al., 2001). The present data should also be considered in the context of therapies intended to reduce immune function and inflammation [e.g., glucocorticoid treatment for rheumatoid arthritis; for a recent review of rheumatoid arthritis treatments including glucocorticoids, see Doan et al. (2005)]. In light of the present data, these treatments may have the potential to exacerbate brain damage in individuals at risk for stroke or other insults. The present research highlights a need for research to determine the effects and time course for heightened risk of certain steroid treatments on susceptibility to brain damage. In conclusion, many conditions involve elevated glucocorticoid levels, and the present research shows that the history of glucocorticoid elevations may be as important as determining current glucocorticoid levels for assessing risk of brain damage. 


\section{References}

Bardgett ME, Taylor GT, Csernansky JG, Newcomer JW, Nock B (1994) Chronic corticosterone treatment impairs spontaneous alternation behavior in rats. Behav Neural Biol 61:186-190.

Bardgett ME, Newcomer JW, Taylor GT (1996) The effects of chronic corticosterone on memory performance in the platform maze task. Physiol Behav 59:1111-1115.

Bodnoff SR, Humphreys AG, Lehman JC, Diamond DM, Rose GM, Meaney MJ (1995) Enduring effects of chronic corticosterone treatment on spatial learning, synaptic plasticity, and hippocampal neuropathology in young and mid-aged rats. J Neurosci 15:61-69.

Coburn-Litvak PS, Pothakos K, Tata DA, McCloskey DP, Anderson BJ (2003) Chronic administration of corticosterone impairs spatial reference memory before spatial working memory in rats. Neurobiol Learn Mem 80:11-23.

Cohen J, Cohen P, West SG, Aiken LS (2003) Applied multiple regression/ correlation analysis for the behavioral sciences, Ed 3. Mahwah, NJ: Erlbaum.

Conrad CD (2006) What is the functional significance of chronic stressinduced CA3 dendritic retraction within the hippocampus? Behav Cogn Neurosci Rev 5:41-60.

Conrad CD, Galea LA, Kuroda Y, McEwen BS (1996) Chronic stress impairs rat spatial memory on the Y-maze, and this effect is blocked by tianeptine pretreatment. Behav Neurosci 110:1321-1334.

Conrad CD, Lupien SJ, McEwen BS (1999a) Support for a biomodal role for type II adrenal steroid receptors in spatial memory. Neurobiol Learn Mem 72:39-46.

Conrad CD, Magariños AM, LeDoux JE, McEwen BS (1999b) Repeated restraint stress facilitates fear conditioning independently of causing hippocampal CA3 dendritic atrophy. Behav Neurosci 113:902-913.

Conrad CD, Jackson JL, Wise L (2004) Chronic stress enhances ibotenic acid-induced damage selectively within the hippocampal CA3 region of male, but not female rats. Neuroscience 125:759-767.

Dachir S, Kadar T, Robinzon B, Levy A (1993) Cognitive deficits induced in young rats by long-term corticosterone administration. Behav Neural Biol 60:103-109.

Dachir S, Robinzon B, Grauer E, Levy A (1995) Nimodipine counteracts corticosterone-induced habituation impairments. Neurobiol Learn Mem 63:241-245

Dallman MF, Bhatnagar S, Via V (2000) Hypothalamo-pituitary-adrenal axis. In: Encyclopedia of stress, Vol 2 (Fink G, ed), pp 468-477. San Diego: Academic.

DeGiorgio LA, Attardi B, Shimizu Y, Ogata M, Volpe BT (2002) 17bEstradiol treatment retards excitotoxic delayed degeneration in substantia nigra reticulata neurons. Brain Res 936:15-20.

de Kloet ER, Joëls M, Holsboer F (2005) Stress and the brain: from adaptation to disease. Nat Rev 6:463-475.

Doan T, Massarotti E (2005) Rheumatoid arthritis: an overview of new and emerging therapies. J Clin Pharmacol 45:751-762.

Endo Y, Nishimura J, Kimura F (1996) Impairment of maze learning in rats following long-term glucocorticoid treatments. Neurosci Lett 203:199-202.

Fitch JM, Juraska JM, Washington LW (1989) The dendritic morphology of pyramidal neurons in the rat hippocampal CA3 area. I. Cell types. Brain Res 479:105-114

Jarrard LE (1989) On the use of ibotenic acid to lesion selectively different components of the hippocampal formation. J Neurosci Methods 29:251-259.

Kleen JK, Sitomer MT, Killeen PR, Conrad CD (2006) Chronic stress im- pairs spatial memory and motivation for reward without disrupting motor ability and motivation to explore. Behav Neurosci 120:842-851.

Landfield PW, Waymire JC, Lynch G (1978) Hippocampal aging and adrenocorticoids: quantitative correlations. Science 202:1098-1102.

Luine VN, Spencer RL, McEwen BS (1993) Effects of chronic corticosterone ingestion on spatial memory performance and hippocampal serotonergic function. Brain Res 616:65-70.

Magariños AM, McEwen BS (1995a) Stress-induced atrophy of apical dendrites of hippocampal CA3c neurons: comparison of stressors. Neuroscience 69:83-88.

Magariños AM, McEwen BS (1995b) Stress-induced atrophy of apical dendrites of hippocampal CA3c neurons: Involvement of glucocorticoid secretion and excitatory amino acid receptors. Neuroscience 69:89-98.

Magariños AM, Orchinik M, McEwen BS (1998) Morphological changes in the hippocampal CA3 region induced by non-invasive glucocorticoid administration: a paradox. Brain Res 809:314-318.

McEwen BS (2000) Effects of adverse experiences on brain structure and function. Biol Psychiatry 48:721-731.

McLaughlin KJ, Gomez J, Baran SE, Conrad CD (2007) The effects of chronic stress on hippocampal morphology and function: an evaluation of chronic restraint paradigms. Brain Res 1161:56-64.

McLay RN, Freeman SM, Zadina JE (1998) Chronic corticosterone impair memory performance in the Barnes maze. Physiol Behav 63:933-937.

Nichols NR, Zieba M, Bye N (2001) Do glucocorticoids contribute to brain aging? Brain Res Rev 37:273-286.

Ramos-Remus C, González-Castañeda RE, González-Perez O, Luquin S, García-Estrada J (2002) Prednisone induces cognitive dysfunction, neuronal degeneration, and reactive gliosis in rats. J Invest Med 50:458-464.

Reagan LP, McKittrick CR, McEwen BS (1999) Corticosterone and phenytoin reduce neuronal nitric oxide synthase messenger RNA expression in rat hippocampus. Neuroscience 91:211-219.

Sapolsky RM (1985) Glucocorticoid toxicity in the hippocampus: temporal aspects of neuronal vulnerability. Brain Res 359:300-305.

Sapolsky RM (2005) The influence of social hierarchy on primate health. Science 308:648-652.

Sapolsky RM, Pulsinelli WA (1985) Glucocorticoids potentiate ischemic injury to neurons: therapeutic implications. Science 229:1397-1399.

Sapolsky RM, Packan DR, Vale WW (1988) Glucocorticoid toxicity in the hippocampus: in vitro demonstration. Brain Res 453:367-371.

Seyle H (1984) The stress of life, revised edition. New York: McGraw-Hill.

Stein-Behrens B, Mattson MP, Chang I, Yeh M, Sapolsky R (1994) Stress exacerbates neuron loss and cytoskeletal pathology in the hippocampus. J Neurosci 14:5373-5380.

Uno H, Tarara R, Else JG, Suleman MA, Sapolsky RM (1989) Hippocampal damage associated with prolonged and fatal stress in primates. J Neurosci 9:1705-1711.

Watanabe Y, Gould E, McEwen BS (1992a) Stress induces atrophy of apical dendrites of hippocampal CA3 pyramidal neurons. Brain Res 588:341-345.

Watanabe Y, Gould E, Cameron HA, Daniels DC, McEwen BS (1992b) Phenytoin prevents stress- and corticosterone-induced atrophy of CA3 pyramidal neurons. Hippocampus 2:431-436.

Woolley CS, Gould E, McEwen BS (1990) Exposure to excess glucocorticoids alters dendritic morphology of adult hippocampal pyramidal neurons. Brain Res 531:225-231.

Wright RL, Lightner EN, Harman JS, Meijer OC, Conrad CD (2006) Attenuating corticosterone levels on the day of memory assessment prevents chronic stress-induced impairments in spatial memory. Eur J Neurosci 24:595-605. 